\title{
Joint parameter estimation and Cramer-Rao bound analysis in ground-based forward scatter radar
}

\author{
Tao Zeng ${ }^{1}$, Cheng $\mathrm{Hu}^{1 *}$, Mikhail Cherniakov², Chao Zhuo ${ }^{3}$ and Cong Mao ${ }^{1}$
}

\begin{abstract}
Forward scatter radar (FSR) has potential applications such as target detection, classification, and recognition. The success of these issues depends on the accuracy of parameter estimation. Many parameter estimation methods for air-based FSR have been given, but cannot directly be applied in the ground-based ones for the different system functions. The received signal in ground-based FSR depends on the target's electrical size and trajectory, which are unknown a priori. It is impossible to construct an optimal reception with accurate reference function in practical situations. An adaptive method of parameter estimation is therefore proposed in this article, which includes the construction of reference function and the two-dimensional parameter estimation. Furthermore, the Crammer-Rao bounds of estimation accuracy are obtained via the analytical derivation, which can be in turn utilized to determine the estimation step in the algorithm. Finally, the effectiveness of the algorithm is shown using both simulated and experimental data.
\end{abstract}

\section{Introduction}

No matter in the modern or future electronic warfare, radar is continuously playing a key role. Historically, bistatic radar is the earliest radar system applied in the warfare field. Only after the substantiation of a pulse method of radar and the development of antenna switches, more convenient monostatic radars were developed and therefore impeded the development of bistatic radar [1,2]. However, in the modern electronic warfare, due to the advent of the fast development of technology, new threats such as electronic interference, low altitude penetration, stealth weapon, and anti-radiation missiles have to be faced and dealt with. The conventional monostatic radar system has been distressed into dilemma. Radar community revisited the potential of the bistatic system due to its potential to deal with the above issues, and then numerous of hardware and software for the bistatic radars have been proposed, investigated, constructed and built up.

Forward scatter radar (FSR) is an extreme bistatic radar configuration, which is generally defined with the bistatic

\footnotetext{
* Correspondence: hucheng.bit@gmail.com

'Department of Electronic Engineering, Beijing Institute of Technology, Beijing 100081, China

Full list of author information is available at the end of the article
}

angle around $170^{\circ}-180^{\circ}$ [3], i.e., the target is sensed close to the radar baseline. The basic principles of FSR can be found in Willis [2] and Chernyak [3,4]. The main difference compared with the conventional radars (both monostatic and bistatic) lies in its target scattering nature. In the forward scattering (FS) area, the radar utilizes the diffraction effects rather than the reflection effects of the electromagnetic wave to capture the target's information, and therefore brings some winning properties compared with conventional ones. The target can be considered as a secondary antenna which has the silhouette of the target. The gain of this secondary antenna is directly related to the target FS Cross-Section (CS) and is independent from the target material [5]. Therefore, such systems are robust to stealth technology and benefit for target detection. Another peculiarity is that the received signal has little phase and amplitude fluctuation. All FS EM wave components are traveling toward the receiving antenna in phase, and the signal could coherently be integrated over a long period of time, which will in return introduces an excellent frequency resolution. As a result, FSR has unique potential in automatic target recognition [6]. In 2000, the great Shadow Inverse Synthetic Aperture theory was proposed by Chapurskiy and Sablin [7], which 
makes significant contribution to target classification and recognition based on FSR system.

Till now, most existing publications dedicated to FSR studies focus on the airborne issues. In the air-based FSR system, the velocity of the air target is high [8], and the length of the baseline is far larger than the target size [9], furthermore the clutter background is relatively small [10]. Therefore, the instantaneous Doppler frequency and angle of arrival can effectively be obtained via Doppler filter and multi-beam antenna, and then based on the nonlinear relationship between target coordinate and Doppler frequency as well as angle of arrival, the approximate coordinate estimates are obtained with numerical solution [8-10], but the methods of air targets detection and tracking cannot be used in the groundbased FSR due to the different system behaviors and target motion characteristics.

The ground-based network of FS Micro Radars (FSMR) has been considered for target detection and automatic recognition in $[3,11-16]$. It has been demonstrated by means of both analytical and experimental studies that ground targets crossing the baseline can reliably be detected. While in these practical ground-based FSR systems, the sensors are positioned nearly on the ground; the operational range is restricted to hundreds of meters due to the local horizon and landscape peculiarity as well as power budget constraints. All these make it impossible to use the mono-pulse antenna or the array antenna to sense the target. So, the angle of arrival cannot be achieved directly. Moreover, the velocity of the ground target is much lower than the air target, the Doppler frequency will not change enough to allow the Doppler filter extract the Doppler shift (the velocity of the target). The conventional signal processing method for the air target detection and parameter estimation cannot be applied in the ground-based system.

In this article, we consider the method of target detection and parameter estimation in the ground-based FSR system. The radars work under the single carrier Continuous Wave mode and have no capability of range resolving. This means that this radar is only able to detect moving targets. Because the direct signature (from transmitter to receiver directly) is far stronger than the moving target signature, using the envelope detector or phase detector, we can extract the signature of moving target (beat frequency). This signature will occur in the background of clutter including foliage and vegetation, thermal and atmospheric and possibly industrial noise and so forth. The latter could be viewed as Additive White Gaussian Noise (AWGN). Therefore, we will only consider the case of target detection against AWGN under the assumption that a target is following a nearly linear trajectory. Furthermore, numerous observations of ground targets confirm that the linear trajectory assumption is correct for practical cases.
Moreover, foliage clutter can be approximated as a narrowband Gaussian process [17-21], and the statistic characteristics in FSMR have empirically been estimated via the experimental data [18-20], thus the optimal detection procedure against clutter background in FSMR includes a whitening filter. Hence, the assumption that the target is detected against AWGN is also true for clutter presence.

Whereas the optimal signal processing algorithms in the presence of AWGN are well known for traditional radars [10], they have to be modified for FSMR, within the bound of classic linear matched filtering (correlation) theory. In this article, we will present an improved algorithm based on the method proposed in [22]. The algorithm is developed primarily for moving target detection, where the two-dimensional parameter (the velocity of the target and the baseline crossing point) estimations are the subsidiary but very important function of this algorithm. We first study the received signal of our system. Due to the symmetry of FSMR system configuration (shown in Figure 1), the envelope and phase signature of the received signal are nearly symmetrical and can be approximated as a double-sided chirp signal with a modulated envelope. This naturally occurring doublesided chirp signal is well known in radar system analysis [10]. We can expect this signal compressed at the optimal filter output, and hence get an enhanced resolution for the targets following in a convoy. To achieve this end, an appropriate reference function is required for optimal reception. But, in contrast to conventional radar, where the reference function is the delayed and frequency shifted transmitted waveform, the received signal in FSR depends on the targets electrical size and trajectory which are unknown a priori. That is because the particularity of the ground-based FSR systems which have been mentioned before. As a result, in practical situations, it is impossible to obtain the accurate reference function at the reception side and adaptive method of optimal parameter estimation is therefore proposed. Many motion parameters of the target, such as the velocity, the orientation, the baseline crossing point, and the acceleration, affect the nature of the received signal. For this reason, taking into consideration the practical situation and to simplify the complexity and cut off the computation cost of the algorithm, two influential parameters among them are considered in the proposed algorithm, namely the velocity and the baseline crossing point. In the groundbased FS area, the target will not have much complex motion, and therefore the impact of the acceleration can be neglected. The proposed algorithm is robust to the orientation of the target, which is to say even if the angle is not equal to $90^{\circ}$, we can also estimate the true radical velocity and obtain a good compression result. We also give the Crammer-Rao bound of the estimation accuracy, which can be in turn utilized to determine the estimated 


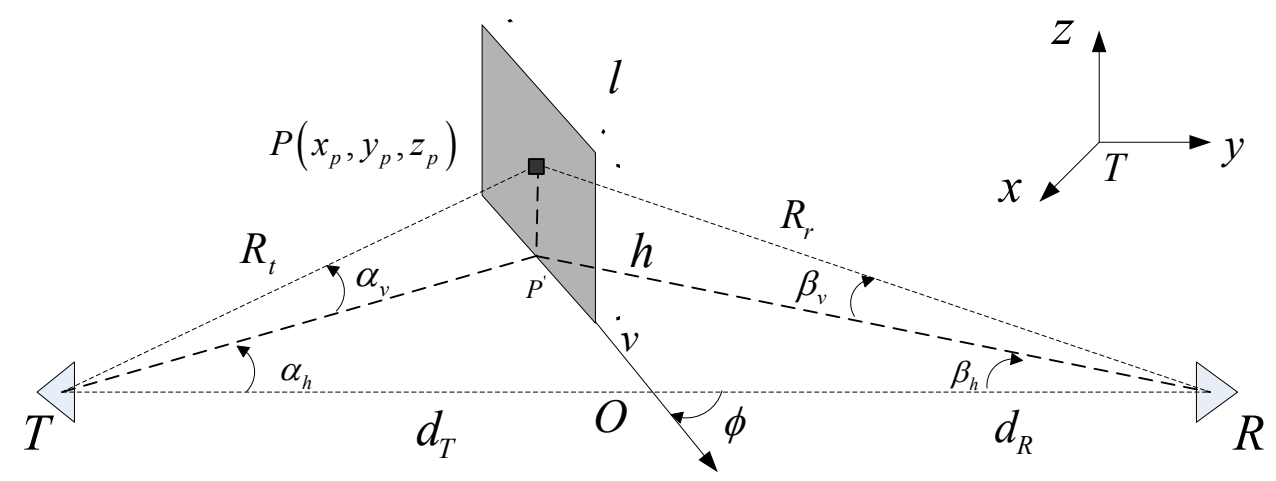

Figure 1 The moving target topology of FS radar.

step of the parameters. Simulation results as well as the experimental data have been used to verify the correctness and the effectiveness of our method.

This article is organized as follows: The signal model will be presented in Section 2. The signal processing algorithm, including signal-to-noise ratio (SNR) maximization and the joint parameter estimation, will be proposed in Section 3. The Crammer-Rao bounds of our method as well as the simulation and experimental results are given in Section 4. Finally, the conclusion is drawn in Section 5.

\section{Signal model of ground moving target in FSR}

In this section, the signal model of FSR ground moving target will be given. Figure 1 shows the topology of the FSR configuration for moving target. The transmitting and receiving antennas have an equal elevation $h_{A}$. The target has the size of $l * h, v$ is the target motion speed, $O$ is the crossing point at the baseline, $R_{t}$ and $R_{r}$ are the range of T-Target (transmitter to target) and $\mathrm{R}$-Target (receiver to target), respectively, $d_{T}$ and $d_{R}$ are the baseline range of $T O$ and $O R$ correspondingly. On the transmitter side, the azimuth and elevation diffraction angle are $\alpha_{h}$ and $\alpha_{v}$, and similarly on the receiver side, we have $\beta_{h}$ and $\beta_{v}$. In short, the relative position of the transmitter, target, and the receiver thoroughly determines the topology of the FSR configuration.

Disregarding the possible interference, clutter, and noise, the signal at the input of the receiving antenna can be presented as a sum of the target echo and the leakage signal [17]:

$$
u_{r}(t)=U_{L} \cos \left(\omega_{0} t+\varphi_{0}\right)-U_{t g}(t) \cos \left(\omega_{0} t+\varphi_{D}(t)+\varphi_{x}\right)
$$

where $U_{L}$ is the magnitude of leakage signal, $\omega_{O}$ is the transmitted carrier frequency, $\phi_{0}$ is the transmitted initial phase. $U_{t g}(t)$ is the magnitude of the target echo, which depends on the shape and location of the target. The minus sign is generated by the negative gain of the shadow beam. $\phi_{D}(t)$ is the Doppler phase which is formed by the Doppler frequency. $\phi_{x}$ is an unknown phase introduced in the FS process. When the leakage signal power is far greater than the target echo power, let the signal in (1) go through the envelope detector, after removing the direct current (DC) component, the target echo magnitude and the Doppler phase can both be extracted. The output can be expressed as

$$
S_{r}(t)=-U_{t g}(t) \cos \left(\varphi_{D}(t)+\varphi_{x}-\varphi_{0}\right)
$$

Using the Kirchhoff's method and Babine's principle for solving the diffraction problem, the phase difference $\phi_{x}-\phi_{0}$ is equal to $90^{\circ}[2]$. Thereby the resultant received signal can be rewritten as

$$
S_{r}(t)=U_{t g}(t) \sin \left(\varphi_{D}(t)\right)=U_{t g}(t) \sin \left(2 \pi f_{d}(t) \cdot t\right)
$$

According to (3), the received target signal consists of two parts, one is the target magnitude $U_{t g}(t)$, and the other is the target Doppler signal $\sin \left(2 \pi f_{d}(t) \cdot t\right)$. Compared with the Doppler signal, the magnitude will not change much during the observation time, and it is largely determined by the propagation mechanism and target scattering model rather than the motion parameter of the target. More details about the characteristic of magnitude can be looked up in [17-21]. On the contrary, the Doppler signal changes much more rapidly with the moving of the target. It is mainly determined by the motion parameter, and is the focus in our algorithm. So, a detailed analysis about the Doppler signal will be presented here.

According to the 3D FSR geometry in Figure 1 and the definition of Doppler signal, we have the target Doppler signal as

$$
f_{d}=-\frac{1}{\lambda} \frac{\mathrm{d}\left(R_{t}(t)+R_{r}(t)\right)}{\mathrm{d} t}=-\frac{\mathrm{d} R_{t}(t)}{\lambda \mathrm{d} t}-\frac{\mathrm{d} R_{r}(t)}{\lambda \mathrm{d} t}
$$

where $d\left(^{*}\right)$ denotes the differential operator. Here, we assume that the target moves in a uniformly linear form. According to Figure 1, we have 


$$
\left\{\begin{array}{l}
R_{t}(t)=\frac{\sqrt{\left(v t \cos \phi+d_{T}\right)^{2}+(v t \sin \phi)^{2}}}{\cos \alpha_{v}} \\
R_{r}(t)=\frac{\sqrt{\left(v t \cos \phi-d_{R}\right)^{2}+(v t \sin \phi)^{2}}}{\cos \beta_{v}}
\end{array}\right.
$$

Then, we have the differentiations as

$$
\left\{\begin{array}{l}
\frac{\mathrm{d} R_{t}(t)}{\lambda \mathrm{d} t}=\frac{v \cos \left(180-\phi-\alpha_{h}(t)\right)}{\lambda \cos \alpha_{v}} \\
\frac{\mathrm{d} R_{r}(t)}{\lambda \mathrm{d} t}=\frac{v \cos \left(\phi-\beta_{h}(t)\right)}{\lambda \cos \beta_{v}}
\end{array}\right.
$$

In the ground-based FSR system, the elevation angles $\alpha_{v}, \beta_{v}$ are very small and can be regarded as a constant approaching to zero. Then, the Doppler frequency can be simplified as

$$
f_{d}(t)=\frac{2 v}{\lambda} \sin \left(\frac{\alpha_{h}(t)+\beta_{h}(t)}{2}\right) \cdot \sin \left(\phi+\frac{\alpha_{h}(t)-\beta_{h}(t)}{2}\right)
$$

Here, we use the substitutions as

$$
\left\{\begin{array}{l}
\cos \left(\alpha_{h}(t)\right)=\frac{v t \cos \phi+d_{T}}{\sqrt{\left(v t \cos \phi+d_{T}\right)^{2}+(v t \sin \phi)^{2}}}, \sin \left(\alpha_{h}(t)\right)=\frac{v t \sin \phi}{\sqrt{\left(v t \cos \phi+d_{T}\right)^{2}+(v t \sin \phi)^{2}}} \\
\cos \left(\alpha_{v}(t)\right)=\frac{v t \cos \phi-d_{R}}{\sqrt{\left(v t \cos \phi-d_{R}\right)^{2}+(v t \sin \phi)^{2}}}, \sin \left(\alpha_{v}(t)\right)=\frac{v t \sin \phi}{\sqrt{\left(v t \cos \phi-d_{R}\right)^{2}+(v t \sin \phi)^{2}}}
\end{array}\right.
$$

In (7), we can see that the Doppler frequency depends on the target velocity, the wavelength of the transmitted signal, and the target horizontal diffraction angle. When the target crosses the baseline, namely that $\alpha_{h}=\beta_{h}=0^{\circ}$, the Doppler frequency in (7) equals to zero. Using this point, we can estimate the target crossing moment in the time-frequency domain. To see how the Doppler signal and the target magnitude behave in the real case, simulation and experimental results are given below.
In Figure 2, two typical experiment scenarios are shown. The first scenario is the stadium of University of Birmingham, the baseline length (from $\operatorname{Tr}$ to $\mathrm{Re}$ ) is $50 \mathrm{~m}$, three experiment frequencies at 69,151 , and $433 \mathrm{MHz}$ are used. The target model is a man with the dimension of $1.75 \mathrm{~m} \times$ $0.65 \mathrm{~m}$ (height $\times$ width), the target moves perpendicularly to the baseline and crosses the baseline at the midpoint with an approximately constant velocity. The environment is relatively clear and broad compared with the second one. Trees and buildings are far from the Tr and Re, thus the clutter is low and clear target echo could be seen (shown in Figure 3a, b). The second experiment scenario is the grass land near to the building, the experiment frequency is $69 \mathrm{MHz}$, the experiment baseline length is also $50 \mathrm{~m}$, the target model is human with the dimension 1.65 $\mathrm{m} \times 0.5 \mathrm{~m}$ (height $\times$ width), the target keeps the constant velocity to cross the baseline midpoint. In the second experiment, the environment is bush, a lot of trees are around the $\operatorname{Tr}$ and $\operatorname{Re}$ as well as the baseline, but because of the clutter immunity of low carrier frequency, the clear target echo could be seen as well (shown in Figure 3c). In addition, the receiver has a low pass filter $(20 \mathrm{~Hz})$ to reduce the noise and high pass filter $(0.1 \mathrm{~Hz})$ to reject the clutter, these filters will result in the slight distortion of target echo. In order to compare the results of experimented data and simulated data, in our simulation, all the parameters are the same with the experiment parameters (including the filter parameters).

Figure 3a-c shows the comparison of the simulated signal and experimental signal at different carrier frequencies. In Figure 3a, the simulated signal has nearly the same Doppler oscillation with the experimental signal, which verifies the correctness of (7). The higher the carrier frequency is, the higher the Doppler oscillation is. As Figure 3a-c illustrates, the target magnitude

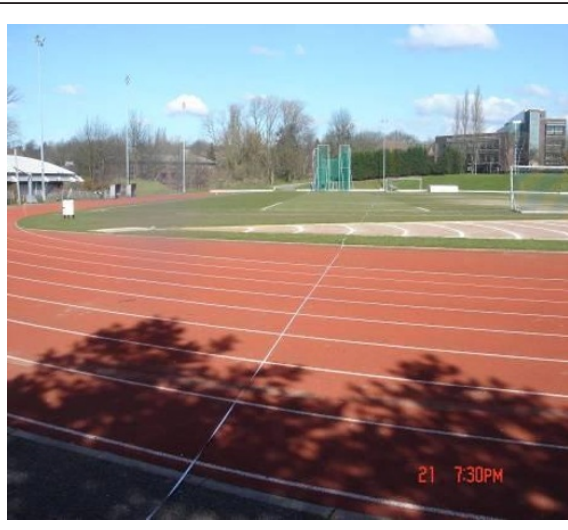

(a) Experiment scenario 1

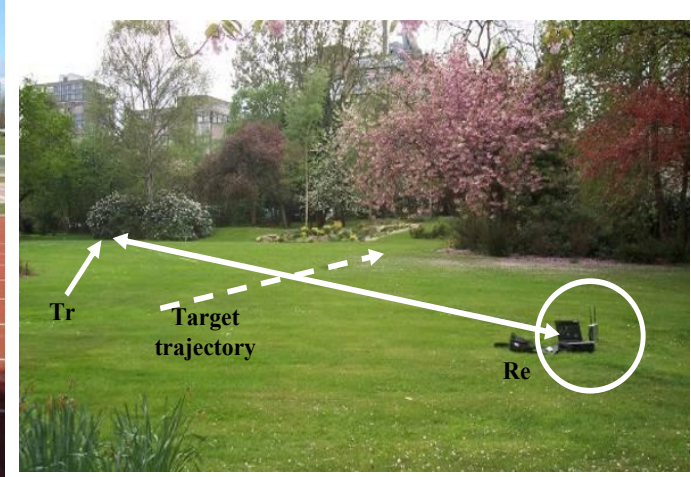

(b) Experiment scenario 2

Figure 2 Experiment scenario configuration. (a) Experiment scenario 1. (b) Experiment scenario 2. 
(a) Simulated and experimented signal at $151 \mathrm{MHz}$

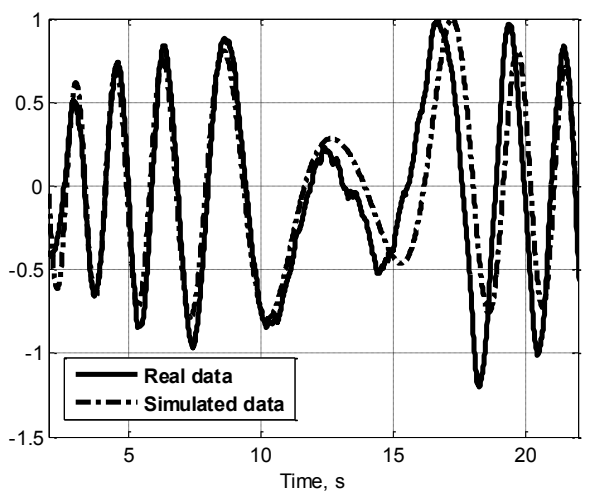

(b) Simulated and experimented signal at $433 \mathrm{MHz}$

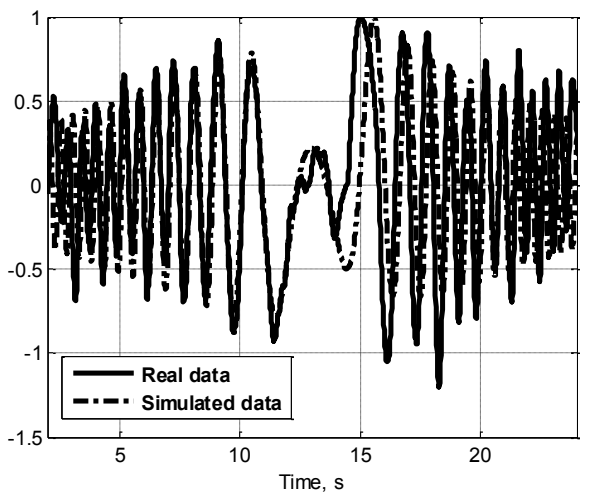

(c) Simulated and experimented signal at $69 \mathrm{MHz}$

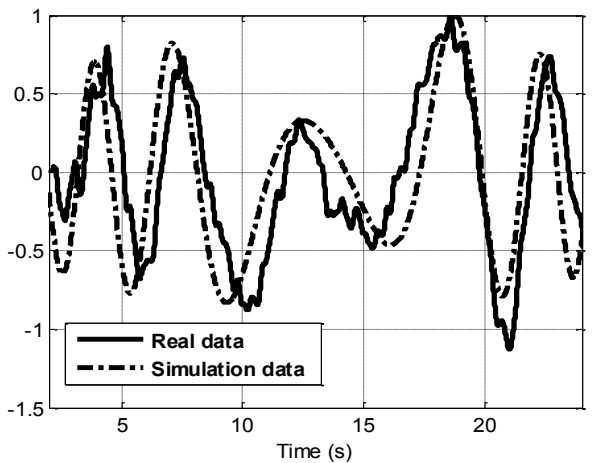

Figure 3 The comparison of simulated signal and experimented signal at different carrier frequency. Simulated and experimented signal at (a) $151 \mathrm{MHz}$, (b) $433 \mathrm{MHz}$, (c) $69 \mathrm{MHz}$. behaves more like a window function without much variation. The fast oscillating phase signal has the signature of a double-sided chirp form. The phase signal obviously shows more close relationship with the target motion parameter and could certainly provide more accurate estimation. In the following discussion, we will approximate the target magnitude with a rectangular window function, which is just used to determine the observation interval.

\section{General signal processing algorithm}

In this section, a joint parameter estimation method will be given, which is an improvement of the method given in [22]. In the classical processing algorithm proposed in [22], three different assumptions have been made, namely that the target's motion direction is normal to the baseline, the target crosses the baseline at the midpoint, and the motion of the target is uniformly linear without acceleration. For the velocity direction angle assumption, it is reasonable because the velocity estimation algorithm is very robust to the velocity direction angle. For the no-acceleration assumption, it is very reasonable as well because the interested ground target (human or car or tank) has very small or no acceleration over the observation time (only a few seconds), the small acceleration only results in the asymmetry of compression side-lobe, does not affect the velocity estimation (shown in [23]). For the midpoint assumption, in the proper operation area $\left(d_{T} / L\right.$ around 0.5 ), we can obtain the correct velocity estimation and good compression results; outside this operation area, the performance of velocity estimation and target compression deteriorates a lot. However, in practical case, it is impossible to guarantee that the target crosses the baseline at midpoint. Moreover, the target crossing position affects the Doppler signal a lot and then causes the deviation of velocity estimation. Therefore, we need a new algorithm to adapt this case, what is to say, we want to estimate the target crossing position and velocity at the same time.

Before estimating the target crossing position and target velocity, we should represent the received signal under the AWGN background, we have

$$
x(t)=S_{r}\left(t, v, d_{T}\right)+n(t), \quad-\frac{T_{\mathrm{obs}}}{2} \leq t \leq \frac{T_{\mathrm{obs}}}{2}
$$

Note that we use $S_{r}\left(t, v, d_{T}\right)$ here to represent $S_{r}(t)$ in (3), for that we assume only the three variables $t, v, d_{T}$ determine the change of Doppler frequency $f_{d}$, and then further determine the change of the received signal $S_{r}$. That is to say, except for the natural variable $t$, the received signal is not only determined by the target velocity, but also by the target crossing position. This is obviously a nonlinear estimation of two variables. 
According to the classical estimation theory [23], log of likelihood ratio function of the signal under the AWGN in (9) can be expressed as

$$
\begin{aligned}
& \ln \Lambda\left[x(t), v, d_{T}\right]=\frac{2}{N_{0}} \int_{-\frac{T_{o b s}}{2}}^{\frac{T_{o b s}}{2}} x(t) S_{r}\left(t, v, d_{T}\right) d t \\
& -\frac{1}{N_{0}} \int_{-\frac{T_{o b s}}{2}}^{\frac{T_{o b s}}{2}} S_{r}^{2}\left(t, v, d_{T}\right) d t
\end{aligned}
$$

Based on (10), we can implement the estimation of velocity and crossing point. To find the maximum of (10), there are two equivalent ways. One way is to find the local maximization by using the conditional equations

$$
\left\{\begin{array}{l}
\left.\int_{-\frac{T_{\mathrm{obs}}}{2}}^{\frac{T_{\mathrm{obs}}}{2}}\left[x(t)-S_{r}\left(t, v, d_{T}\right)\right] \frac{\partial S_{r}\left(t, v, d_{T}\right)}{\partial v} d t\right|_{v=\hat{v}_{m l}}=0 \\
\left.\int_{-\frac{T_{\mathrm{obs}}}{2}}^{\frac{T_{\mathrm{obs}}}{2}}\left[x(t)-S_{r}\left(t, v, d_{T}\right)\right] \frac{\partial S_{r}\left(t, v, d_{T}\right)}{\partial d T} d t\right|_{d_{T}=\hat{d}_{T} m l}=0
\end{array}\right.
$$

A possible way to implement (11) is the block diagram shown in Figure 4.

From (11), because of the high nonlinearity, we cannot obtain the analytical expression of $\hat{v}_{m l}$ and $\hat{d}_{T m l}$, we can only obtain the numerical solution from the block diagram in Figure 4. But this is a time-consuming process.

According to (10), the second term is nearly a constant representing the SNR. The basic operation on the received data consists of generating the first term in (10) as a function of $v$ and $d_{T}$. Then, we can rewrite the (10) as

$$
l\left(v, d_{T}\right)=\int_{-\frac{T_{o b s}}{2}}^{\frac{T_{o b s}}{2}} x(t) S_{r}\left(t, v, d_{T}\right) d t
$$

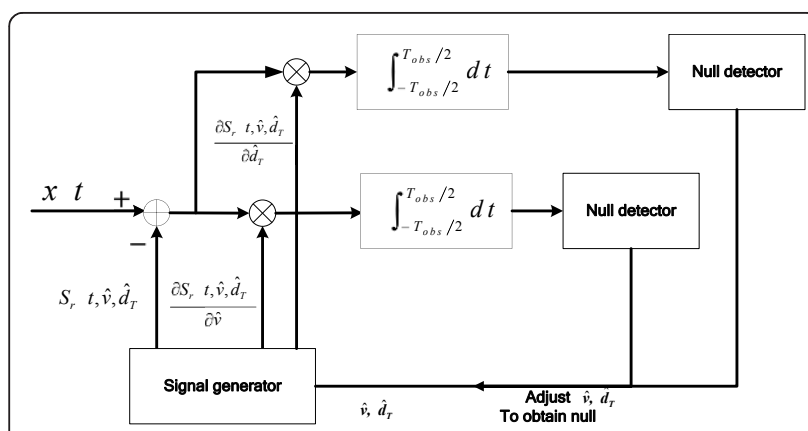

Figure 4 The optimal estimator structure (velocity and crossing position estimation).
Based on (12), an alternate approach equivalent to (10) is to divide the range into increments of length $\Delta v$, $\Delta d$ and perform the parallel processing operation shown in Figure 5 for discrete values of $v$ and $d_{T}$

$$
\begin{aligned}
& v_{1}=0+\frac{\Delta v}{2}, \quad d_{T 1}=d_{T 0}+\frac{\Delta d}{2} \\
& v_{2}=0+\frac{3 \Delta v}{2}, \quad d_{T 2}=d_{T 0}+\frac{3 \Delta d}{2} \\
& \left\{\begin{array}{l}
M=\left[\frac{V_{m}}{\Delta v}+\frac{1}{2}\right] \\
P=\left[\frac{L-2 \cdot d_{T 0}}{\Delta d}+\frac{1}{2}\right](13)
\end{array}\right. \\
& v_{M}=0+\left(M-\frac{1}{2}\right) \Delta v, d_{T P}=d_{T 0}+\left(P-\frac{1}{2}\right) \Delta d
\end{aligned}
$$

where $L$ is the baseline length, $d_{T 0}$ is the starting range from target to transmitter, $M$ and $P$ are the channel numbers of the filter bank.

The operation structure shown in Figure 5 is an equivalent implementation of (11). The corresponding parameters $\left(v_{m}, d_{T p}\right)$ with the maximum output are the final estimated results. The values of $\Delta v, \Delta d$ in (13) are very important parameters in our algorithm. If the intervals are too long, the estimated results will deviate from the real values a lot and reduce the estimation accuracy. On the other hand, if the intervals are too short, it will dramatically increase the computation cost. Therefore, selecting appropriate incremental intervals is very important, and the Crammer-Rao bounds of the estimation are naturally good references. Using the theoretically best accuracy, i.e., the Crammer-Rao bound, the estimated step can guarantee a good estimation as well as an appropriate computation cost.

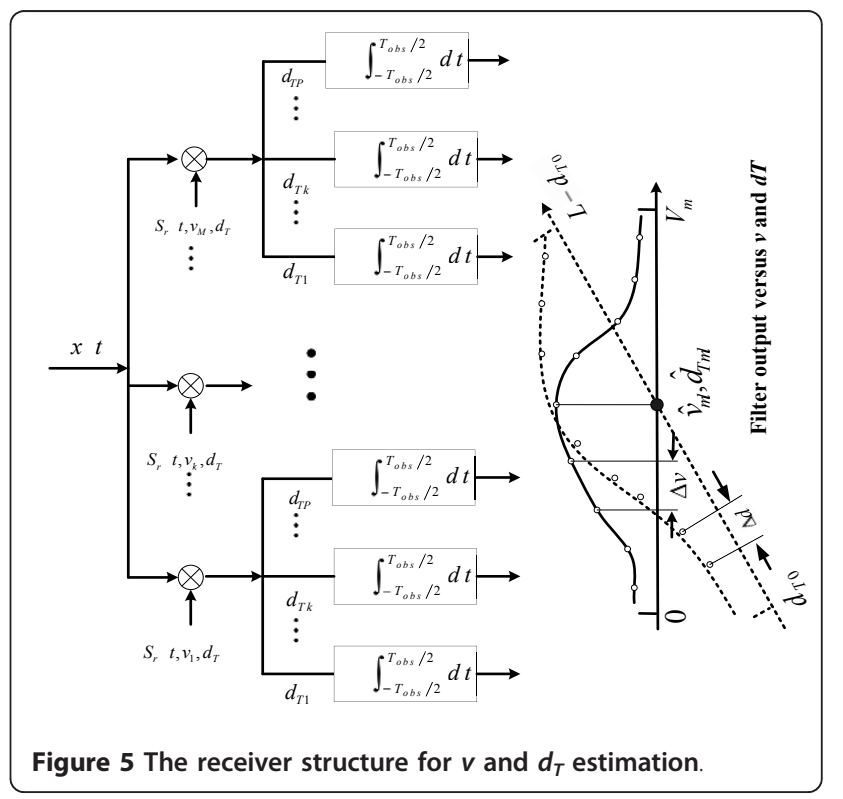




\section{The Crammer-Rao bound}

A possible way to accomplish our algorithm is given in Figure 5. We expect that we choose the correct interval in our preliminary processing and the final accuracy would be closely approximated by the Crammer-Rao low bound (CRLB). The Crammer-Rao low bound can be obtained by inverting the Fisher Information Matrix $F$ whose elements are the expectation of the second derivative (with respect to $v$ and $d_{T}$ ) of the Log likelihood function [23]. The Fisher Information Matrix can be written as

$$
F=\left[\begin{array}{ll}
A & B \\
B & C
\end{array}\right]=\left[\begin{array}{l}
E\left[\frac{\partial^{2} \ln \Lambda\left(t, v, d_{T}\right)}{\partial v^{2}}\right] E\left[\frac{\partial^{2} \ln \Lambda\left(t, v, d_{T}\right)}{\partial v \partial d_{T}}\right] \\
E\left[\frac{\partial^{2} \ln \Lambda\left(t, v, d_{T}\right)}{\partial v \partial d_{T}}\right] \\
E\left[\frac{\partial^{2} \ln \Lambda\left(t, v, d_{T}\right)}{\partial d_{T}^{2}}\right]
\end{array}\right]
$$

Thus, it is not difficult to obtain the CRLB:

$$
\mathrm{CRLB}=F^{-1}=\frac{1}{A C-B^{2}}\left[\begin{array}{cc}
C & -B \\
-B & A
\end{array}\right]
$$

The variations of $A, B$, and $C$ in the case of $d_{T}=0.5 L$ are shown in Figure 6a, and the variations of $A, B$, and $C$ in the case of $d_{T}=0.2_{\mathrm{L}}$ are shown Figure 6b. For a human target, the observation time is usually more than $25 \mathrm{~s}$, and it can be known that $A \gg C>B$, namely that $A C \gg B^{2}$ (shown in Figure 7), so we think the effect of $B$ can be ignored when calculating the CRLB. That is to say, the CRLB can be simplified as

$$
\mathrm{CRBL}=F^{-1} \approx \frac{1}{A C}\left[\begin{array}{lc}
C & -B \\
-B & A
\end{array}\right]=\left[\begin{array}{ll}
A^{-1} & -B /(A C) \\
-B /(A C) & C^{-1}
\end{array}\right]
$$

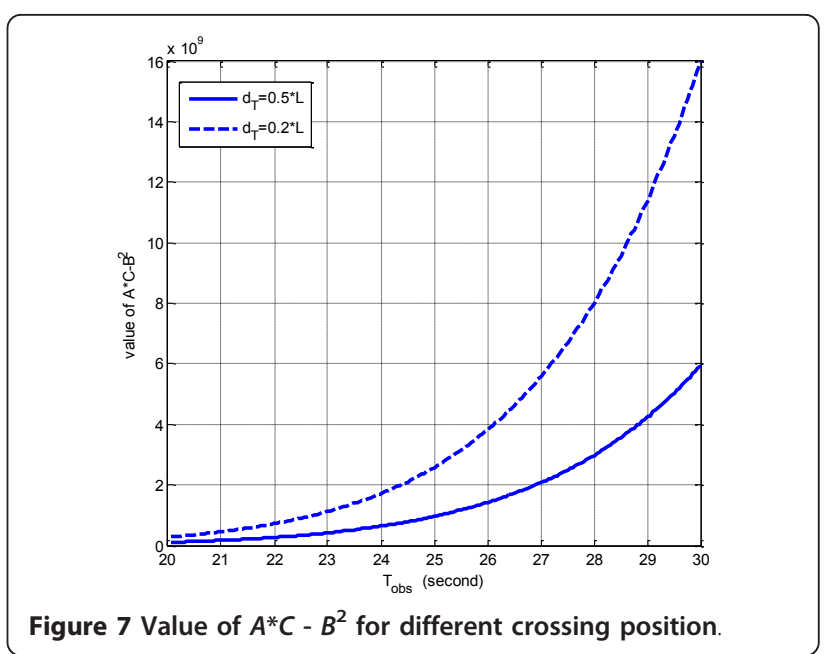

For the non-random variable, we differentiate (10) and take the expectation, then we have

$$
\begin{aligned}
& E\left[\frac{\partial^{2} \ln \Lambda\left[x(t), v, d_{T}\right]}{\partial v^{2}}\right] \\
& =\frac{2}{N_{0}}\left\{E \int_{-\frac{T_{o b s}}{2}}^{\frac{T_{o b s}}{2}}\left[x(t)-S_{r}\left(t, v, d_{T}\right)\right] \frac{\partial^{2} S_{r}\left(t, v, d_{T}\right)}{\partial v^{2}} d t-E \int_{-\frac{T_{o b s}}{2}}^{\frac{T_{o b s}}{2}}\left[\frac{\partial S_{r}\left(t, v, d_{T}\right)}{\partial v}\right]^{2} d t\right\}
\end{aligned}
$$

$E\left[\frac{\partial^{2} \ln \Lambda\left[x(t), v, d_{T}\right]}{\partial d_{T}^{2}}\right]$

$=\frac{2}{N_{0}}\left\{E \int_{-\frac{T_{o b s}}{2}}^{\frac{T_{o b s}}{2}}\left[x(t)-S_{r}\left(t, v, d_{T}\right)\right] \frac{\partial^{2} S_{r}\left(t, v, d_{T}\right)}{\partial d_{T}^{2}} d t-E \int_{-\frac{T_{o b s}}{2}}^{\frac{T_{o b s}}{2}}\left[\frac{\partial S_{r}\left(t, v, d_{T}\right)}{\partial d_{T}}\right]^{2} d t\right\}$

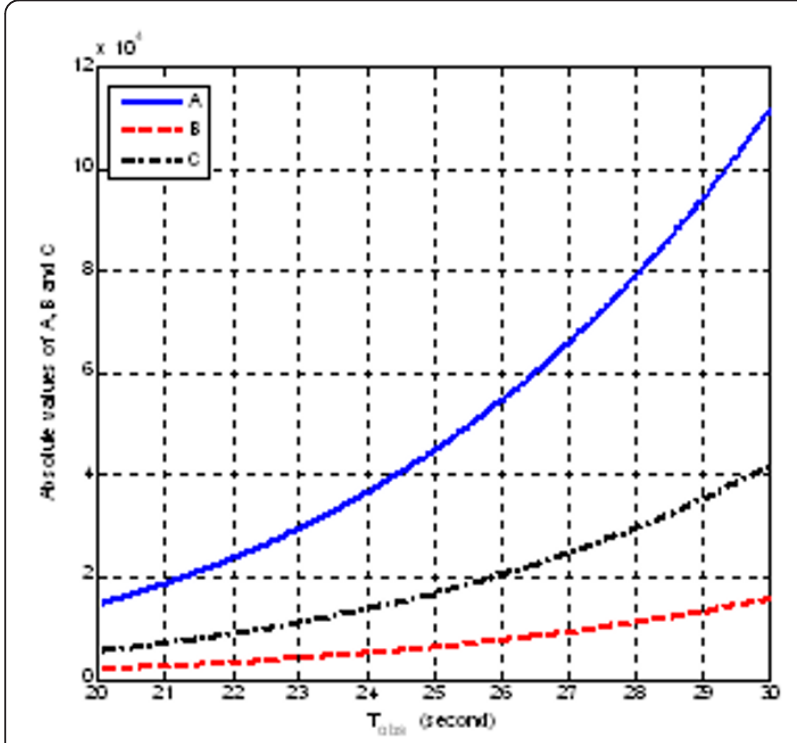

(a) $d T=0.5 L$

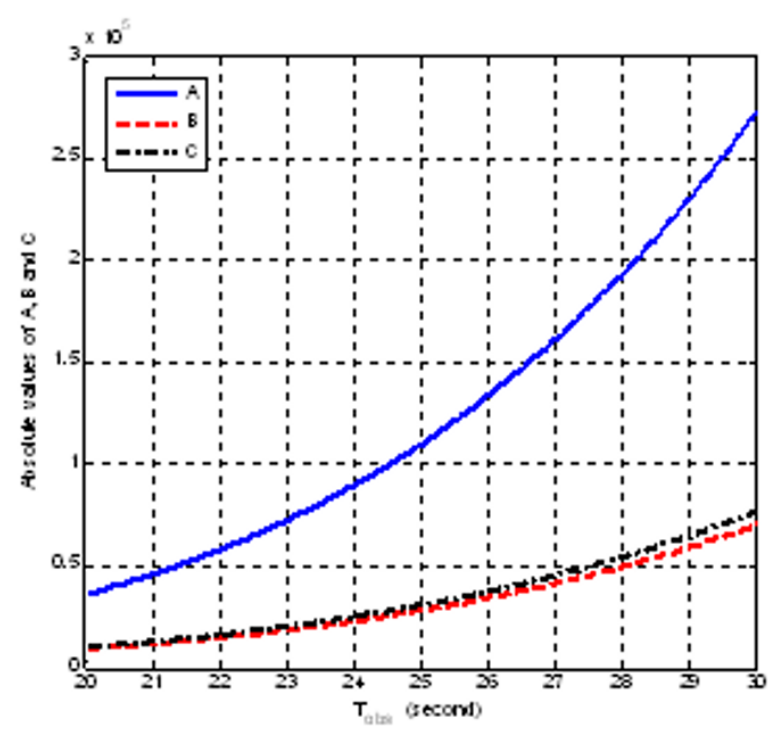

(b) $d T=0.2 L$

Figure 6 Absolute value of $\boldsymbol{A}, \boldsymbol{B}$ and $\boldsymbol{C}$. (a) $d_{T}=0.5 \mathrm{~L}$; (b) $d_{T}=0.2 \mathrm{~L}$. 
where $E[*]$ means the expectation operator. In the first term of (17) and (18), we observe a factor that

$$
E\left[x(t)-S_{r}\left(t, v, d_{T}\right)\right]=E[n(t)]=0
$$

It will make the first integral term to be zero. In the second term of (17) and (18), there are no random quantities, and therefore the expectation operation gives the integral itself. In addition, the partial derivative of the signal can be rewritten as

$$
\begin{aligned}
& \frac{\partial S_{r}\left(t, v, d_{T}\right)}{\partial v} \approx \frac{\partial\left(U_{t g} \sin \left(2 \pi f_{d} t\right)\right)}{\partial v} \\
& \quad \approx U_{t g} \cdot \frac{2 \pi v L t^{2}}{\lambda d_{T} \cdot\left(L-d_{T}\right)} \cdot \cos \left(\pi \frac{v^{2} L}{\lambda d_{T} \cdot\left(L-d_{T}\right)} t^{2}\right)^{(20)} \\
& \frac{\partial S_{r}\left(t, v, d_{T}\right)}{\partial d_{T}} \approx \frac{\partial\left(U_{t g} \sin \left(2 \pi f_{d} t\right)\right)}{\partial d_{T}} \\
& \quad \approx U_{t g} \cdot \frac{\pi v^{2} t^{2}\left(2 d_{T}-L\right)}{\lambda d_{T}^{2} \cdot\left(L-d_{T}\right)^{2}} \cdot \cos \left(\pi \frac{v^{2} L}{\lambda d_{T} \cdot\left(L-d_{T}\right)} t^{2}\right)^{(21)}
\end{aligned}
$$

Substituting (19)-(21) into (17) and (18), we have

$$
\begin{aligned}
\gamma_{v}^{2} & =\operatorname{Var}\left[\hat{v}_{m l}-v\right] \geq \frac{N_{0}}{2 \int \frac{T_{\text {obs }}}{-\frac{T_{\text {obs }}}{2}}\left[\frac{\partial S_{r}\left(t, v, d_{T}\right)}{\partial v}\right]^{2} d t} \\
& \approx \frac{5}{4 \cdot \frac{E_{r}}{N_{0}} \cdot \tan ^{2} \alpha \cdot \frac{\pi^{2} T_{\text {obs }}^{2}}{\lambda^{2}}} \\
\gamma_{d}^{2} & =\operatorname{Var}\left[\hat{d}_{T m l}-d_{T}\right] \geq \frac{N_{0}}{2 \int \frac{T_{\text {obs }}^{2}}{-\frac{T_{\text {obs }}}{2}}\left[\frac{\partial S_{r}\left(t, v, d_{T}\right)}{\partial d_{T}}\right]^{2} d t} \\
& \approx \frac{10}{\frac{E_{r}}{N_{0}} \cdot \frac{\pi^{2}}{\lambda^{2}} \cdot\left(\tan ^{2} \beta-\tan ^{2} \alpha\right)^{2}}
\end{aligned}
$$

Equations (22) and (23) give the analytical expression of the Crammer-Rao bounds for velocity estimation and baseline crossing point estimation. Both of these two estimation accuracies are in proportion to the SNR. The higher the SNR is, the better the estimation accuracies are. As for the velocity estimation accuracy, it is also in proportion to the azimuth angle $\alpha_{h}$ and the observation time $T_{o b s}$. This is because the larger $\alpha_{h}$ and the longer $T_{o b s}$ will lead to a bigger Doppler shift. Meanwhile, a longer wavelength will reduce the sensitivity of the Doppler frequency, and therefore impact the estimation accuracy. For the crossing point estimation, a bigger difference between the $\alpha_{h}$ and $\beta_{h}$ will lead to a bigger difference between the Doppler signals, and therefore results in a better estimation with higher accuracy. As for the wavelength, it has the same conclusion as the velocity estimation. Based on the analytical expression of (22) and (23), simulation results of the Crammer-Rao bounds for the velocity and crossing point estimation are presented in Figure 8, which is under the typical experimental parameter.

From Figure 8, we can see that, when the SNR is 15 $\mathrm{dB}$, the square root of CRLB for the velocity estimation is less than $0.02 \mathrm{~m} / \mathrm{s}$, for the crossing point estimation it is less than $0.5 \mathrm{~m}$. So, the estimated step should be set as $0.02 \mathrm{~m} / \mathrm{s}$ for velocity estimation and $0.5 \mathrm{~m}$ for crossing point estimation (where the target model is human with a velocity of $1 \mathrm{~m} / \mathrm{s}$, the baseline length is $50 \mathrm{~m}$ ). If so, the estimation accuracy of the relative velocity can reach $2 \%$, which can well satisfy the requirement for target recognition.

Noting that in the ground-based FSR system, we do not estimate the velocity parameter and crossing position at the moment of crossing baseline. The velocity and crossing position are estimated via (11) with the whole received signal (double-side chirp signal shown in Figure 3 ). Take the velocity estimation for example, from (13), we can see that the target velocity estimation accuracy depends on the choice of velocity step $\Delta v=v_{i}$ $v_{\mathrm{i}-1}$. If we assume the target's true velocity is $v$, a velocity deviation $\Delta v$ introduces envelope and phase errors to the reference function (double-side chirp signal) used for signal compression. In general, the effect of envelope error is far less than that of phase error. Therefore, the required $\Delta v$ can be determined by the phase error size. As a rule of thumb, taking pi/ 4 as the maximum tolerable phase error, the choice of $\Delta v$ can be written as

$$
\frac{\Delta v}{v} \leq \frac{\lambda}{8 \cdot\left(d_{T}+d_{R}\right) \cdot \tan ^{2} \alpha_{\operatorname{Max}}}
$$

where $\alpha_{\text {Max }}$ is the maximal diffraction angle. We can see that velocity step depends on the carrier frequency, baseline distance, and target velocity, where wavelength and baseline distance are known in advance, the diffraction angle are also preset beforehand and approximately determined by the target FS patterns. Here, we assume, as an example, that baseline distance is equal to $50 \mathrm{~m}, \alpha_{\operatorname{Max}}$ is $30^{\circ}$ (for a human target) and for typical velocity (for a human, $1 \mathrm{~m} / \mathrm{s}$ ) and carrier frequency $(151 \mathrm{MHz})$, the velocity step should be chosen as $0.015 \mathrm{~m} / \mathrm{s}$ at most.

Figure 9 gives the simulation and the experimental result of the signal processing method in this paper. For comparison, in Figure 10, we first present the processing result of the classical method in [22] under the same experimental condition. The target crosses the baseline perpendicularly at a distance of $d_{T}=10 \mathrm{~m}$ from the transmitter. The 
(a) The square root of CRB of velocity estimation ( $T_{o b s}=28 s, \alpha_{h}=30^{\circ}$, human target)

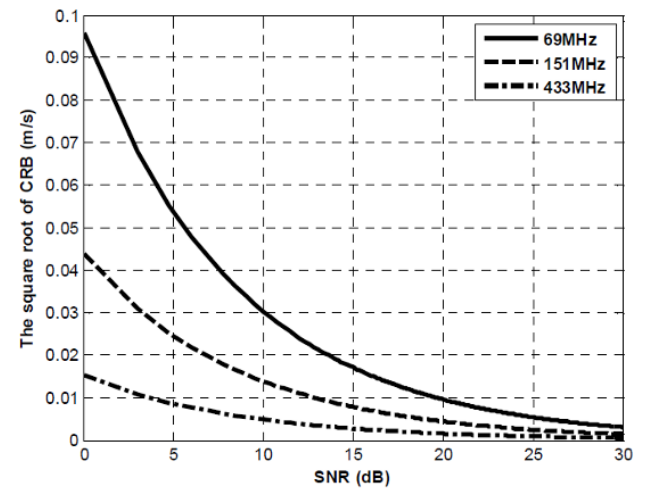

(b) The square root of the CRB of $d_{T}$ estimation ( $d_{T} / L=0.2$ case)

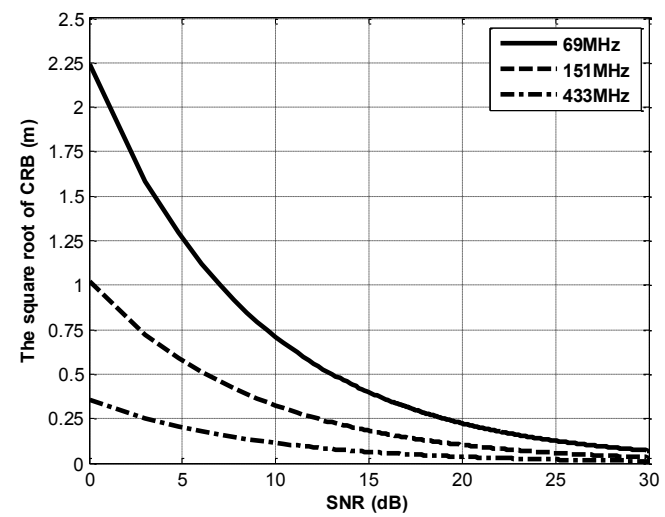

Figure 8 The square root of the CRBs for velocity estimation and $\boldsymbol{d}_{T}$ estimation. (a) The square root of CRB of velocity estimation ( $T_{\text {obs }}=$ $28 \mathrm{~s}, \alpha_{h}=30^{\circ}$, human target). (b) The square root of the CRB of $d_{T}$ estimation ( $d_{T} / L=0.2$ case).

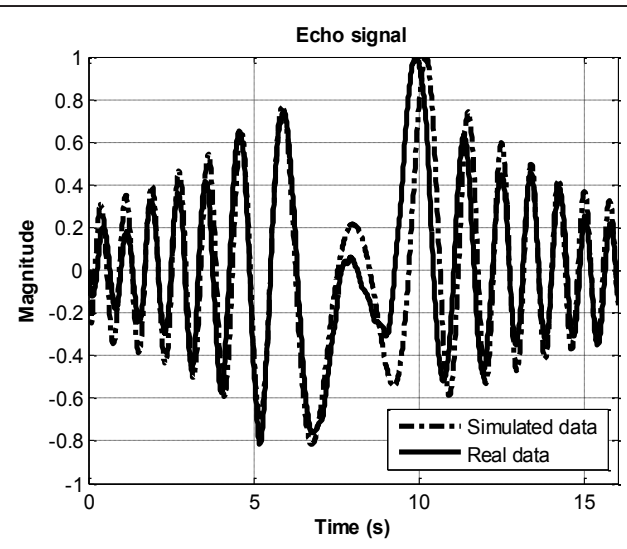

(a) Experimented data and simulated data

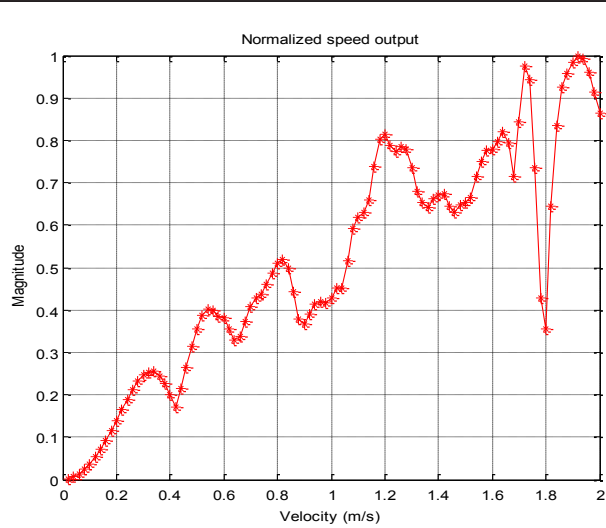

(b) The output of velocity filter bank

Figure 9 The contour of two dimensional filter output ( $\boldsymbol{v}$ and $\boldsymbol{d}_{T}$ output, $\boldsymbol{d}_{T} / \mathbf{L}=\mathbf{0 . 2}$ ). (a) The processed result of simulated data. (b) The processed result of experimented data. 


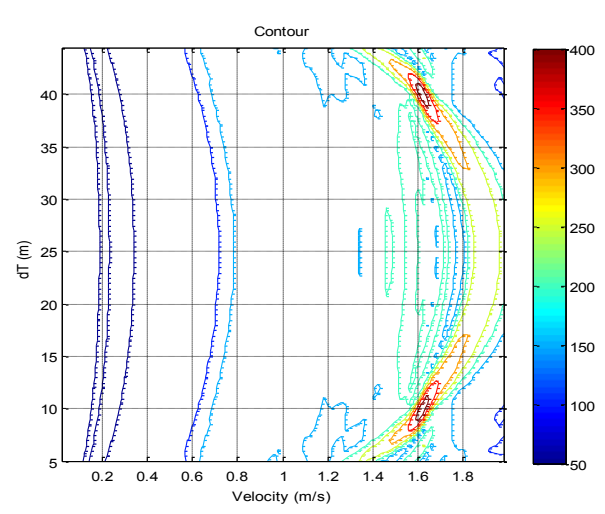

(a) The processed result of simulated data

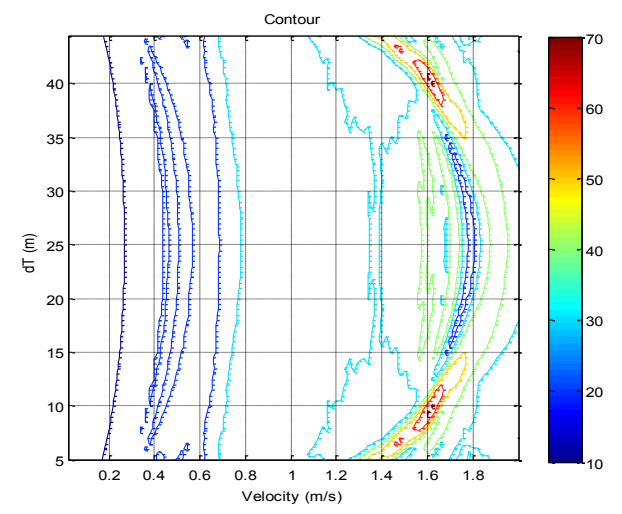

(b) The processed result of experimented data

Figure 10 The experimented data processing result of $d_{T} / L=0.2(151 \mathrm{MHz})$. (a) Experimented data and simulated data. (b) The output of velocity filter bank.

length of the baseline is $50 \mathrm{~m}$. The true velocity of the target is $1.62 \mathrm{~m} / \mathrm{s}$. Figure 10a shows the received signal of the simulation and experimental data. In Figure 10b, it shows the estimated velocity by the classical method is $1.92 \mathrm{~m} / \mathrm{s}$ which deviates from the true value a lot. The deviation percentage is $\frac{1.92-1.62}{1.62} \times 100 \%=18.5 \%$. While in Figure 9, we can see that both the velocity and the crossing point are estimated correctly. The estimated velocity is $1.62 \mathrm{~m} / \mathrm{s}$, the crossing point is estimated at 10 and $40 \mathrm{~m}$.

The simulated and experimental results show that the classical processing algorithm cannot obtain the correct the velocity estimation because of the mismatched target crossing position. Our new algorithm cannot only estimate the velocity, but also estimate the target crossing position correctly, although there is no range resolution in the baseline direction. In addition, the new algorithm extend the operation area, that is to say no matter where is the target crossing position, we can always obtain good estimations of the $v$ and $d_{T}$, contrary to the classical processing algorithm only works in the operation area around $d_{T} / L=0.5$ (crossing near the midpoint).

We should point out that there is an ambiguous crossing position in Figure 8. This is because the reference signal is symmetrical when the constructed reference signal has the distance $d_{T}$ to the transmitter or to the receiver. If we use the netted FSR, it is not difficult to solve the ambiguity problem of crossing position.

\section{Conclusions}

In this article, we have presented a signal processing method for the ground-based FSR system, including the target detection and the joint parameter estimation. It extended the operation area of the classical method.
The estimated parameters are very important for further requirements such as target classification and recognition. The Crammer-Rao bounds for the accuracy of the estimations have also been given and be used to determine the estimated step. The algorithm was tested both on simulated and experimental data, all of the results verify the effectiveness of our algorithm.

\section{Acknowledgements}

This study was supported by the National Natural Sciences Foundation of China (Grant Nos. 61172177, 61032009, 61120106004), and partially supported by the Electro-Magnetic Remote Sensing Defence Technology Centre, established by the UK Ministry of Defence, under Project No. 1-27.

\section{Author details}

'Department of Electronic Engineering, Beijing Institute of Technology, Beijing 100081, China 2University of Birmingham, Edgbaston, B15 2T, UK ${ }^{3}$ School of Electronic Information, Soochow University, Suzhou, Jiangsu 215021, China

\section{Competing interests}

The authors declare that they have no competing interests.

Received: 15 May 2011 Accepted: 11 April 2012 Published: 11 April 2012

\section{References}

1. RJ Boyle, Comparison of monostatic and bistatic bearing estimation performance for low RCS targets. IEEE Trans AES. 30(3), 962-968 (1994)

2. NJ Willis, Bistatic Radar (Technology Service Corporation, Raleigh, 1995)

3. M Cherniakov (ed.), Bistatic Radar: Principles and Practice (Wiley \& Sons, New York, 2007)

4. V Chernyak, Fundamentals of Multisite Radar Systems (Gordon and Breach Science Publishers, NV, 1998)

5. PY Ufimtsev, Comments on the diffraction principles and limitations of RCS reduction techniques. Proc IEEE. 84(12), 1830-185 (1996)

6. Ml Skolnik, Introduction to Radar Systems (McGraw-Hill Book Company, New York, 1980), p. 558

7. VV Chapurskiy, VN Sablin, SISAR: shadow inverse synthetic aperture radiolocation, in Proc Int Radar Conf 2000, Washington, DC, USA, 332-328 (2000)

8. AV Myakinkov, Optimal detection of high-velocity targets in forward scattering radar, in 5th International Conference on Antenna Theory and Techniques, Kyiv, Ukraine, 2005, 345-347 (24-27 May2005) 
9. AB Blyakhman, AG Ryndyk, SB Sidorov, Forward scattering radar moving object coordinate measurement, in The Record of the IEEE 2000 International Radar Conference, Alexandria, VA, 678-682 (7-12 May 2000)

10. DM Gould, RS Orton, RJE Pollard, Forward scatter radar detection, in Radar 2002, Edinburgh, UK, 36-40 (15-17 October 2002)

11. M Cherniakov, R Abdullah, P Jančovič, M Salous, V Chapursky, Automatic ground target classification using FSR, in IEE Proceedings Radar, Sonar and Navigation. 153(5), 427-437 (October 2006). doi:10.1049/ip-rsn:20050028

12. R Abdullah, M Cherniakov, P Jančovič, M Salous, Progress on using principle component analysis in FSR for vehicle classification, in Int Workshop on Intelligent Transportation - WIT, Hamburg, Germany, 7-12 (2005)

13. M Cherniakov, R Abdullah, V Chapursky, Short range forward scattering radar, in Int Radar Conf, 322-328 (October 2004)

14. R Abdullah, M Cherniakov, P Jančovič, Automatic vehicle classification in forward scattering radar, in 1st Int Workshop on Intelligent Transportation WIT, Hamburg, Germany, 7-12 (2004)

15. M Cherniakov, M Salous, M Kostylev, R Abdullah, Analysis of forward scattering radar for ground target detection, in 2nd European Radar Conf., Paris, France, 145-148 (2005)

16. M Cherniakov, M Salous, P Jančovič, R Abdullah, V Kostylev, Forward scattering radar for ground targets detection and recognition, in Defence Technology Conf, Edinburgh, UK, A-12 (2005)

17. V Sizov, M Cherniakov, M Antoniou, Forward scattering radar power budget analysis for ground targets, in IEE Proc Radar sonar and Navigation. 1(6), 437-446 (2007). doi:10.1049/iet-rsn:20060174

18. T Long, C Hu, M Cherniakov, Ground moving target signal model and power calculation in forward scattering micro radar. Sci China Ser F Inf Sci. 52(9) (2009). doi:10.1007/s11432-009-0154-1

19. T Long, C Hu, T Zeng, XL Li, Physical modeling and spectrum spread analysis of surface clutter in forward scattering radar. Sci China Inf Sci. 53, 1-14 (2010). doi:10.1007/s11432-010-4085-7

20. C Hu, T Long, T Zeng, Statistic characteristic analysis of forward scattering surface clutter in bistatic radar. Sci China Inf Sci. 53, 1-12 (2010). doi:10.1007/s11432-010-4119-1

21. VI Kostylev, M Cherniakov, Analysis of the signal model for forward scattering radar in case of a small target, in Proceedings of the 5th European Radar Conference, Munich, Germany, 126-129 (2007)

22. H Cheng, M Antoniou, M Cherniakov, V Sizov, Quasi-optimal signal processing in ground forward scattering radar, in RADAR '08. IEEE, Radar Conference, Roman, Italy, 1-6 (2008)

23. V Trees, L Harry, Detection, Estimation, and Modulation Theory, (John Wiley \& Sons, New York, 1971)

doi:10.1186/1687-6180-2012-80

Cite this article as: Zeng et al.: Joint parameter estimation and Cramer-

Rao bound analysis in ground-based forward scatter radar. EURASIP

Journal on Advances in Signal Processing 2012 2012:80.

\section{Submit your manuscript to a SpringerOpen ${ }^{\circ}$ journal and benefit from:}

- Convenient online submission

- Rigorous peer review

- Immediate publication on acceptance

- Open access: articles freely available online

- High visibility within the field

- Retaining the copyright to your article

Submit your next manuscript at $\gg$ springeropen.com 